\title{
SOBRE LOS CONFLICTOS TERRITORIALES DEL SIGLO XXI. ENTREVISTA A HACKITECTURA.NET
}

\author{
ON THE TERRITORIAL CONFLICTS \\ OF THE 21ST CENTURY. AN INTERVIEW \\ TO HACKITECTURA.NET
}

JOSÉ MARÍA SÁNCHEZ-LAULHÉ**
Universidad de Córdoba

El colectivo Hackitectura.net (2001-2010), por su carácter transgresor y experimental, es reconocido únicamente por instancias minoritarias, pese a haber desarrollado trabajos y exposiciones en centros de referencia. Esta investigación tiene uno de sus ejes en una serie de entrevistas realizadas con los tres miembros del kernel de Hackitectura.net (Sergio Moreno, José Pérez de Lama y Pablo de Soto) en que se intenta contextualizar su obra y ese cambio de paradigma en relación con la digitalización y la globalización que tiene lugar en el cambio de siglo. Sus reflexiones nos permiten proyectarlas sobre los conflictos territoriales contemporáneos.

territorio, espacio digital, software libre, arquitectura, activismo
Recibido: 6 de junio del 2019 / Aprobado: 9 de agosto del 2019 doi: 10.26439/limaq2020.n006.4819

The collaborative group Hackitectura.net (2001-2010), due to its transgressive and experimental nature, is only acknowledged by minority groups, despite having developed works and exhibitions in reference centers. One of the main points of this research consists in a series of interviews conducted with three of the Hackitectura.net core members (Sergio Moreno, José Pérez de Lama and Pablo de Soto). These interviews are aimed at contextualizing their work and the paradigm shift regarding the digitization and globalization taken place at the turn of the century. Their reflections enable us to project them on contemporary territorial conflicts.

territory, digital space, free software,

architecture, activism

\footnotetext{
* Este artículo se elaboró a partir de entrevistas a Sergio Moreno, José Pérez de Lama y Pablo de Soto. Las entrevistas a los miembros de Hackitectura.net se realizaron durante el mes de marzo del 2019, en diferentes encuentros debido a su escasa disponibilidad.
} 


\section{INTRODUCCIÓN}

El mundo real, la emisión abierta y la participación desde distintas geolocalizaciones y su puesta en escena son las claves para redefinir la arquitectura y activar el nuevo espacio público.

Pablo de Soto (Hackitectura.net) en el evento Okupa Futura, Corvera, Asturias, 2003

Esta cita de Pablo de Soto nos sitúa en uno de los primeros eventos de los que formó parte el colectivo Hackitectura.net y preámbulo del primer capítulo de Devenires Cíborg. Arquitectura, urbanismo y redes de comunicación (Pérez de Lama, Universidad de Sevilla, 2006) el único libro que recopila parte de la obra del colectivo. Casi una década después de la descomposición de este colectivo (Sevilla y otros lugares, 2001-2010), esta entrevista sirve como reivindicación de su acción y una mirada a nuestra actualidad por parte de estos autores independientes en la acción sobre lo territorial con el nacimiento de la tercera revolución digital y la globalización. Hackitectura.net es un colectivo informe en el que se puede distinguir un kernel principal formado por Sergio Moreno, alias Chaser (Alicante, 1979); José Pérez de Lama, alias Osfa (Sevilla, 1962) y Pablo de Soto (Gijón, 1978). Sus acciones siempre apostaron por generar recursos perdurables para la comunidad (ya sea en formato software libre, cartográfico o recursos materiales) más allá de la entidad propia del evento donde se produjesen. Aunque su singular obra tiene grandes y conocidos seguidores, el estudio de su trabajo sigue estando recluido en instancias minoritarias.

El concepto de hackitectura surge en el año 2001 como una hipótesis surgida tras unas jornadas de hacktivismo coordinada por Natalie Bookchin y la gente de Nettime ${ }^{1}$ en Los Ángeles, desarrollada posteriormente en forma de artículo: "Si hay hacktivismo, por qué no hay hackitectura" (Pérez de Lama, 2000). Hacia el 2003 el concepto se consolida y el colectivo lo hace suyo en la suma de tres acepciones del término recogidas en Devenires Cíborg... (Pérez de Lama, 2006):

Hackitectura.01: uso de forma no prevista - y generalmente subversivade sistemas arquitectónicos y urbanos.

Hackitectura.02, (hackitecto/a): acción, evento, situación construida del agenciamiento de hackers y arquitectos.

Hackitectura 03: redes sociales + redes telemáticas + espacios/territorios; tácticas de producción espacial vinculadas a los procesos emergentes de las nuevas geografías (rizomáticas, fluidas) de la multitud. (p. 55)

Al ser inseparable de su contexto, Pablo de Soto (PDS) se atreve a explicar, con los códigos actuales, que Hackitectura.net

1 Nettime (ahora en nettime.org) fue una mailing list surgida a mediados de los 90 en la que participaban activistas, artistas y teóricos de los medios digitales como Bruce Sterling o Geert Lovink. 
fue una hipótesis de uso radical de unas tecnologías que en ese momento eran nuevas, vinculadas con una serie de conflictos que estaban sucediendo en nuestro entorno geopolítico. El momento era un momento de ruptura en el que surge internet y unas tecnologías nuevas como el streaming o el wireless y que Hackitectura las toma como sus materiales de construcción para repensar unas prácticas espaciales que prototiparan esas arquitecturas de los flujos. Esos cambios históricos no pasan cada 10 años, pasan cada más. Al unísono tenía lugar un momento activista, un momento de movimientos sociales en el mundo que eran el avance de unas ideas emancipadoras: crear infraestructuras comunes globales, crear medios de comunicación independientes, los movimientos norte-sur, los movimientos de solidaridad con inmigrantes. Hackitectura puso esas cosas en relación: tecnologías nuevas con las que experimentamos y un contexto político de expansión de luchas por nuevos derechos (inmigrantes, ámbito digital de las comunicaciones, software libre...).

\section{PRIMERAS ACCIONES E HIPÓTESIS}

Las primeras acciones de Hackitectura.net surgen desde una superposición de iteraciones realizadas debido a impulsos discontinuos de diversos actores de la ciudad. Estas acaban consolidándose en torno a tres identidades que perduran en el tiempo: la trayectoria y capacidad de conectar elementos heterogéneos de José Pérez de Lama, las capacidades técnicas de Sergio Moreno y las intuiciones suyas sumadas a las de Pablo de Soto. Todos coincidieron en la Escuela de Arquitectura de Sevilla, donde en los años 90 el conflicto sobre el espacio público y la nueva fisonomía de la ciudad estaba muy presente y esa búsqueda de nuevos formatos para reivindicar un modelo de ciudad convivencial impulsó la experimentación inicial. Nos cuenta Sergio (SM):

Una de las primeras cosas la hice con mi compañero de piso. Queríamos hacer un streaming y nos pusimos a averiguar qué había. Se lo comentamos a Osfa y ahí empezamos a hacer streaming hacia México con Fran Ilich $^{2}$. A lo mejor lo seguían dos personas. Una cosa ridícula. A veces lanzábamos la señal y no recibíamos nada. Estábamos ahí... pero era un rollo de flipar con las tecnologías y las cosas que se podían hacer nuevas. A partir de ahí, Pablo contactó con la gente de Riereta (http://riereta.net/) mientras hacíamos streaming cada vez más sofisticado, y fue entonces cuando entramos en contacto con el Pure Data. Como seguíamos con el streaming nos pusimos a investigar cómo mandar señales en directo entre Pure Data para hacer sesiones transformadas en tiempo real. Después entramos en contacto con Lluis Gómez (Hangar) e Yves Degoyon, uno de los desarrolladores de Pure Data. Luego fue cuando dimos el salto al GISS ${ }^{3}$ (donde Sergio fue uno de los principales desarrolladores).

2 Fran llich es un artista visual mexicano con el que José Pérez de Lama coincidió en su estancia en Los Ángeles y Chiapas. Extendió ese contacto a otros equipos andaluces como ZEMOS98, con quienes también trabajó.

3 GISS (Global Independent Streaming Support) es una herramienta aún activa en Giss.tv para la realización de streaming bajo software libre. Pure Data es un lenguaje de programación gráfico enfocado para la creación de música. 
Completa José Pérez de Lama (JPL):

Sí, Pablo estaba muy fascinado con el streaming y Sergio sabía cómo hacerlo. Hicimos una serie de proyecciones por San Luis. Luego vino nuestra acción en el Centre de Cultura Contemporània de Barcelona (CCCB). Interesó el proyecto a su director de entonces, Javier Planas, y allí nos plantamos con todos los equipos prestados por la Universidad de Sevilla - en aquella época este tipo de tecnologías no estaban tan extendidas-. Los equipos de la Universidad estaban muy parados porque no sabían en qué darles uso. Después de Barcelona, llegaron [a] una fiesta tras las manifestaciones contra la guerra debajo del Puente del Alamillo, muy chula, organizada por Pablo y la Cartuja Beta Rave.

El streaming, de esta manera, no era únicamente la resolución de un problema técnico para una mayoría (por la carencia, a finales de los 90, de herramientas de comunicación en video a tiempo real), era experimentar cómo la inserción de estas nuevas capacidades técnicas modificaba las geografías y los territorios. En sus propias palabras, "la construcción de situaciones empleando herramientas digitales que permitieran la apropiación y resignificación de un espacio (urbano)" (Pérez de Lama, 2006, p. 57). Y gestionarlo aprovechando la redistribución de roles que produjo internet en el cambio de siglo, con un proceso de divulgación y una adaptación hasta conseguir una versión de software libre útil para la experimentación y el uso profesional:

Los eventos consistían en conectar a la red los espacios que se intervienen, con el mayor ancho de banda posible, usar estas conexiones para, en tiempo real, recibir emisiones desde distintas geolocalizaciones, a la vez que se emite en la red, también en directo -streaming/webcasting-, lo que sucede localmente. Este rizoma de datos se proyectaba en el propio espacio donde sucedía el evento dotándolo así de una segunda piel fluida, real/virtual e interactiva. A la vez, construimos una interfaz interactiva que permitía experimentar el evento, en directo y en diversos modos en la red. (Pérez de Lama, 2006, p. 59)

\section{INDYMEDIA Y FADAIAT. CONSTRUCCIÓN DE UNOS MODOS DE HACER}

“También, en aquella época, estábamos intentando montar un nodo de Indymedia”, nos recuerda JPL. Indymedia era una red global y horizontal de medios de comunicación independiente surgida en la contracumbre de Seattle en 1999. A partir de ahí empezaron a crearse nuevos nodos autónomos, generando una red que compartía recursos y conocimientos. "Indymedia fue pionera en la web 2.0", expresa José Pérez de Lama, quien estuvo presente en los primeros pasos de los nodos de Indymedia Los Ángeles e Indymedia Chiapas. En el sur se hizo una propuesta alternativa a lo habitual con Indymedia Estrecho, saltando de los límites geográficos a las lógicas geopolíticas. "Para ello, la asamblea fundacional, aparte de correos electrónicos, fue una ruta: desde Sevilla primero fuimos a Jerez y luego fuimos a Málaga para consolidar el nodo”. Finalmente, este territorio incluiría el sur de la península ibérica, 
el Magreb y las Islas Canarias arrancando con una fiesta de presentación en 2003. Junto a Hackitectura participaban del nodo otros grupos de activistas, artistas y hackers como ZEMOS98, la Casa de la Paz, la Casa de Iniciativas, el colectivo Aljaima o la Red de las Dos Orillas.

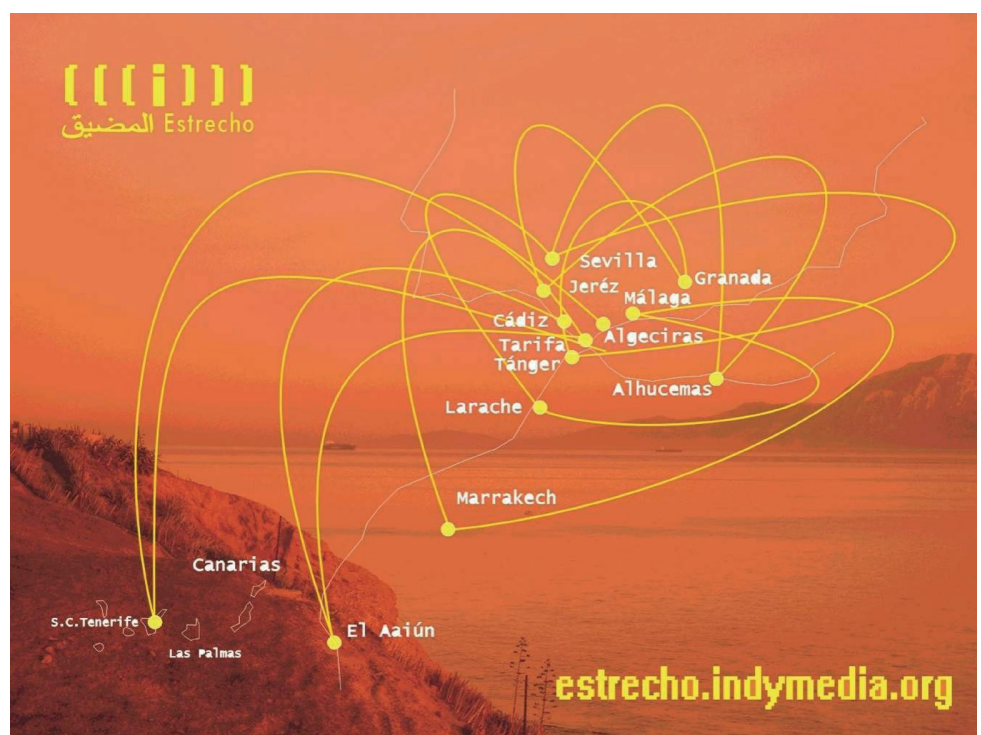

\title{
Figura 1.
}

Mapa fundacio-

nal de Indymedio

Estrecho

Fuente: José

Luis Tirado y

José Pérez de

Lama (2003)

Ser un colectivo con una base pequeña pero que participaba de movimientos y redes globales se soportaba mediante equilibrios precarios como nos cuenta PDS:

\begin{abstract}
Teníamos una base en Sevilla - la casa de Sergio fue muy importante- y después había mucha pendulación a otros lugares. Osfa y yo viajábamos más, relacionándonos con redes activistas (Red NoBorder de Schneider ${ }^{5}$, Indymedia, hackmeetings) del estado español y europeo. Sergio era fundamental por la coordinación y gestión del proyecto. Capturábamos la imaginación de muchos hackers y de muchos activistas. Con ellos construíamos los proyectos. Éramos capaces de liderar a nivel de propuesta una generación (española y europea) que quería experimentar de manera radical qué se puede hacer en la frontera, qué se puede hacer con la tecnología, qué se puede hacer con internet... siempre al límite de lo imposible, de la ciencia ficción. Conseguíamos un think tank con un nivel de radicalidad y de praxis muy experimental.
\end{abstract}

A nivel interno, el kernel del colectivo (Sergio, José y Pablo) eran los elementos de continuidad, pero cuando se concretaban las diferentes acciones, se dilataba

4 Indymedia Estrecho estuvo activa entre 2003-2013. Para más información, aunque la web original ya no sea funcional, ir a este enlace: http://hackitectura.net/osfavelados/indymedia estrecho/index.html

5 Para más información sobre la red NoBorder: http://www.noborder.org/index.php.html 
y desestructuraba adquiriendo con el paso del tiempo un formato menos centralizado y donde el desarrollo de las distintas áreas estaba abierto a las incorporaciones, desvaneciéndose la asignación de autorías, como nos dice JPL:

Pablo era más de la parte conceptual y artística. Sergio de la parte conceptual y técnica. Luego, cuando hicimos la cooperativa WWB de la parte de producción, con Laura Hernández también, yo estaba más en los movimientos sociales y tenía la parte más teórica. Sergio ponía los inputs y las limitaciones técnicas a las ideas que surgían. Y cuando digo "Sergio" incluyo a otros programadores que colaboraban con nosotros. (Lluis, Jonhattan, Rama, Pedro Soler, Jaume)

A partir del evento "Reu03", un encuentro enfocado a arte emergente, las acciones de Hackitectura.net crecen en recursos y necesidades presupuestarias. Por un lado, permite que ciertas investigaciones se consoliden, pero por otro significa que son atravesadas en mayor grado por las instituciones que las amparan o que financian los procesos. Desde la institución les hablaban de cómo participar de la acción pública, de cómo les iba a dar promoción a sus iniciativas, y que necesitaban ayuda y confianza mutua "a lo que respondíamos que de ellos solo necesitábamos el dinero, no la ayuda ni la promoción", confiesa JPL. Eso generaba desencuentros periódicos como nos comenta PDS:

Tuvimos apoyo los primeros años de la Universidad Internacional de Andalucía (UNIA), donde estaba el área de Arte y Pensamiento coordinada por Mar Villaespesa y era una comisaria muy abierta a las cuestiones políticas más instigantes y prácticas artísticas más experimentales. El grupo BNV Producciones, que coordinaba esta área, nos daba cierta confianza y apoyo. No mucho, pero suficiente para publicar la cartografía del Estrecho y la primera edición de Fadaiat. Había en Andalucía esos nichos que ya hacían un trabajo sólido en materia política y artística. La relación fue un poco tensa. Para hacer posible esos proyectos de Hackitectura, teníamos que tensionar la parte de contenidos, la parte de los recursos... pero sí que nos dieron cobertura desde UNIA-Arte y Pensamiento. También del grupo de Florian Schneider que tenía financiación europea, ayudando en Fadaiat. Después, algunos actores de ámbito más de izquierda, como el concejal de IU del ayuntamiento de Tarifa quien auspiciaba Fadaiat, o cuando hicimos el proyecto de la Central Nuclear de Valdecaballeros, un juez de paz fue quien nos dio cobertura. Siempre nos encontramos con algunos elementos particulares a los que conseguimos cautivarles para generar una situación que era difícil de pronosticar previamente pero que capturaba su imaginación.

Concluye JPL sobre esa tensión entre la participación del activismo más ortodoxo y el trabajo junto a instituciones artísticas: "Éramos muy salvajes y el encuentro era complicado con el circuito de arte. Después de estos conflictos nos quedamos un poco apartados de estos colectivos".

En cualquier caso, se concretaron varios de los proyectos que más repercusión tienen en la obra de Hackitectura.net y que les abrieron las puertas a 
aventuras más ambiciosas. Uno de esos proyectos es Fadaiat: "Es el nombre que se da en árabe coloquial a las antenas parabólicas. Quiere decir, literalmente, nave espacial" (Pérez de Lama, 2006, p. 143). Fadaiat es un proyecto de conexión de un territorio que las instituciones tratan de compartimentar y enfrentar con diferentes estrategias, y que corresponde al área del estrecho de Gibraltar. También es una proyección de la crisis del modelo que desde la Unión Europea nos imponen, con la frontera y la militarización de este territorio como símbolos. En este proyecto adquiere cuerpo propio la Cartografía del Estrecho de Gibraltar, recientemente recuperada en la exposición colectiva El Mediterráneo como Frontera comisariado por la propia Mar Villaespesa y Joaquín Vázquez de BNV Producciones ${ }^{6}$.

JPL comenta que la cartografía era una técnica de trabajo que relacionaba cosas aparentemente heterogéneas que se enlazaban 'haciendo mapa', como dirían Deleuze y Guattari. Para él hacer mapa es conectar cosas que en principio eran diferentes para producir nuevos acontecimientos, que en su caso era, por un lado el mundo de la arquitectura con el de las tecnologías y el activismo, y por otro lado conectar con las personas. Ahí es cuando aparece la influencia de los situacionistas, que tenían relación con la globalización y las tecnologías. De acuerdo con los situacionistas los cuerpos en el espacio generan una ciudad distinta a la urbe oficial y más convencional.

\section{WIKIPLAZA. TCS2, SITUATION ROOM, WATER 4 BITS, MAPPING THE COMMONS}

Nos cuenta JPL que en aquel momento estaban por delante de casi todos. No ellos, sino la red de la que participaban gracias a que, en aquel momento, la barrera de entrada era mucho más baja: "parecía que iba a cambiar el mundo". Había una mayor inversión de tiempo personal ante esa perspectiva de cambio que, posteriormente, la entrada de las grandes corporaciones y la crisis fue mermando.

A partir del encuentro TCS2 se crea la cooperativa WWB como un paso más en la consolidación del colectivo. Tuvo lugar en Valdecaballeros dentro de un programa de la Junta de Extremadura sobre la Sociedad de la Información, entonces un proyecto muy importante para Extremadura que había desarrollado el proyecto Línex ${ }^{7}$.

En ese trayecto decidieron realizar una serie de encuentros, uno de los cuales comisionamos nosotros. Quisimos hacer el encuentro en la Central Nuclear abandonada de Valdecaballeros, pero no nos dejaron y al final realizamos una cúpula geodésica con la colaboración de los Straddle, que ya habían participado en Fadaiat.

6 Realizado en el Centro de las Artes de Sevilla en el año 2016: http://icas-sevilla.org/exposicionel-mediterraneo-como-frontera-realidad-y-representacion-en-el-cas/

7 Para más información sobre el proyecto Línex: https://es.wikipedia.org/wiki/GnuLinEx 
Además, participó del encuentro mucha más gente de la creciente red de hackitectura: Ewen Chardronnet, Brian Holmes, Bureau d'Etudes, Clausthome, Exyzt $^{8}$.

En esa época se superpusieron proyectos y hubo entradas y salidas constantes de colaboradores en el equipo. Una de las más estables, Laura Hernández, impulsó el capítulo de producción, como nos recuerda SM:

\begin{abstract}
A Laura la conocí en la Performance Telemática de Los Pajaritos, una idea que surge tras tomarnos un café después de una charla en Zemos98, año 2005 o así. Hicimos un streaming entre Arizona y Los Pajaritos, que lo montamos en una hora. Y se dio ese momento mágico. Fue increíble. ¡Tras ese momento íbamos a muerte! A partir de ahí se montó el proyecto Paso junto a Salud López. Todo era GISS — mucho más depurado-, Pure Data, un montón de ordenadores, live y performance. Un proyecto muy hackitectura. [...] Laura Hernández estuvo al $100 \%$ en la producción del proyecto Wikiplaza. Yo lo hacía todo con ella los siguientes tres años.
\end{abstract}

A raíz de ese trabajo, la Performance Colectiva Internacional PASO (Sevilla, diciembre del 2006) conectó vía streaming coreógrafos y bailarines de Brasil, Arizona y Sevilla en tiempo real. Desde una mirada artística, aliada con las nuevas tecnologías, se desarrolla un discurso global y crítico sobre los derechos humanos y sobre su incumplimiento.

La Wikiplaza es una instancia urbana de las cuestiones que venía trabajando el colectivo en sus trabajos previos en torno al potencial emancipatorio de las tecnologías de la información y la comunicación, reforzando la autoorganización y la autonomía, la producción de recursos del común y la multiplicación de las singularidades (Moreno, 2011, p. 8). La plasmación en el medio urbano implica un esfuerzo en la búsqueda de personas y colectivos que hasta ahora no se habían sentido interpelados por las acciones previas de Hackitectura.net.

La Wikiplaza fue un trabajo conceptual muy interesante, ya que pasamos de todas estas cosas sueltas, y más que trabajaban los movimientos sociales, a la Plaza de las Libertades (proyecto que se bloqueó). Decidimos convertir esas ideas en una especie de matriz de módulos independientes que tenía mucho que ver con el pensamiento cartográfico. Los módulos eran abiertos, aunque costaba encontrar versiones estables. La idea era que se pudieran añadir más módulos. Algunos como David Juárez, de Straddle, lo hicieron. La Wikiplaza tenía la ambición de convertirse en un nombre común más que un proyecto asociado a Hackitectura.

nos dice JPL. Parte del proyecto se presentó en el TCS2 y le gustó a Ewen Chardronnet quien les invitó a realizarlo en París - constituyendo la primera instancia - en el 2008. Dicho proyecto estaba formado por seis módulos principales: Mille Plateaux, un estudio audiovisual para emitir audio y vídeo por

8 Todos estos compañeros de aventuras de Hackitectura.net han recibido reconocimientos a nivel europeo de diversa índole en materia de arte, activismo o acción urbana. 
internet; Situation Room, que luego se expandiría en el proyecto realizado en Asturias; Open MediaLab, que es otro de los fetiches constantes en la obra del colectivo; Mapping Lab, sobre procesos de cartografía sobre software libre; Urban Screen, una pantalla urbana sobre la propia cúpula geodésica; $y$, por último, Inventar Jugando, desarrollado para la instancia en Figueres y dirigido por Susanna Tesconi para acercar a niños y padres al objeto extraterrestre que constituía la Wikiplaza.

Como nos dice SM, en París las condiciones externas condicionaban la idea de espacio público, abierto y autogestionado:

Estuvo muy bien el proyecto de la Wikiplaza pero tuvo condicionantes de "hasta qué hora puede estar abierto, qué se puede hacer aquí, lo que no se puede hacer aquí"... un poco delicado. De hecho, militarizaron la plaza con ese extraño concepto de hacer un prototipo de espacio público y estar rodeado de policías con pistolas. El ayuntamiento decidió montar una seguridad descomunal que no permitía usar la plaza por la noche... París fue la prueba de todos los sistemas: plató de TV, talleres, una programación con un trabajo de red social bastante interesante. Pero en un contexto tan difícil no acabó de estar totalmente liberado su ser. Había un ambiente generalizado de control... La que más me gustó fue la instancia de Figueres. Con una escala más adecuada. Se hicieron las mismas cosas con una producción más distribuida en la que participaron colectivos más heterogéneos. Nosotros coordinábamos - Laura y yo-, pero había otros muchos encargados de otras partes. Era más Wikiplaza. De las cosas más bonitas que hicimos en las Wikiplaza fueron los talleres con los niños que dirigió Susanna Tesconi: construyendo cosas, pintando la calle... ese ambiente de: “ivamos a tomar la calle!”.

En los últimos años de actividad del colectivo, surgen una serie de proyectos que cierran o avanzan en líneas que estaban en marcha: Situation Room, Water 4 Bits y Mapping the Commons. Situation Room es una experiencia de orden artístico/investigador sobre las salas de situación, tras ser la propuesta seleccionada por LABoral Centro de Arte de Gijón. Recuperan la memoria del proyecto realizado por Salvador Allende en Chile por las posibilidades que ofrece para "regular la red como un organismo vivo, donde la autonomía de las células es compatible con el funcionamiento general del organismo" (De Soto, 2010), en lugar de ser una estrategia del control y la punición. Water 4 Bits (W4B), que fue incluida dentro del programa de la Bienal de Arte Contemporáneo de Sevilla, fue una propuesta que generó cierta polémica, puesto que un colectivo de creadores locales había generado una plataforma anti-BIACS que representaba un modelo cultural y artístico distinto al que ellos defendían. Siguiendo la línea de trabajo de Hackitectura, el elemento expuesto servía como interfaz de un trabajo más a largo plazo de experimentación y de puesta en crisis del modelo territorial, en este caso con las ruinas ballardianas de la EXPO'92 como telón de fondo. En concreto, el inutilizado y parcialmente inundado Pabellón de Europa, al cual se realizaron una serie de visitas prospectivas para pequeños grupos. Una serie de sensores en el espacio físico del pabellón se iban representando en el espacio virtual realizado en Open Simulator, alternativa libre a Second Life. Nos explica PDS: 


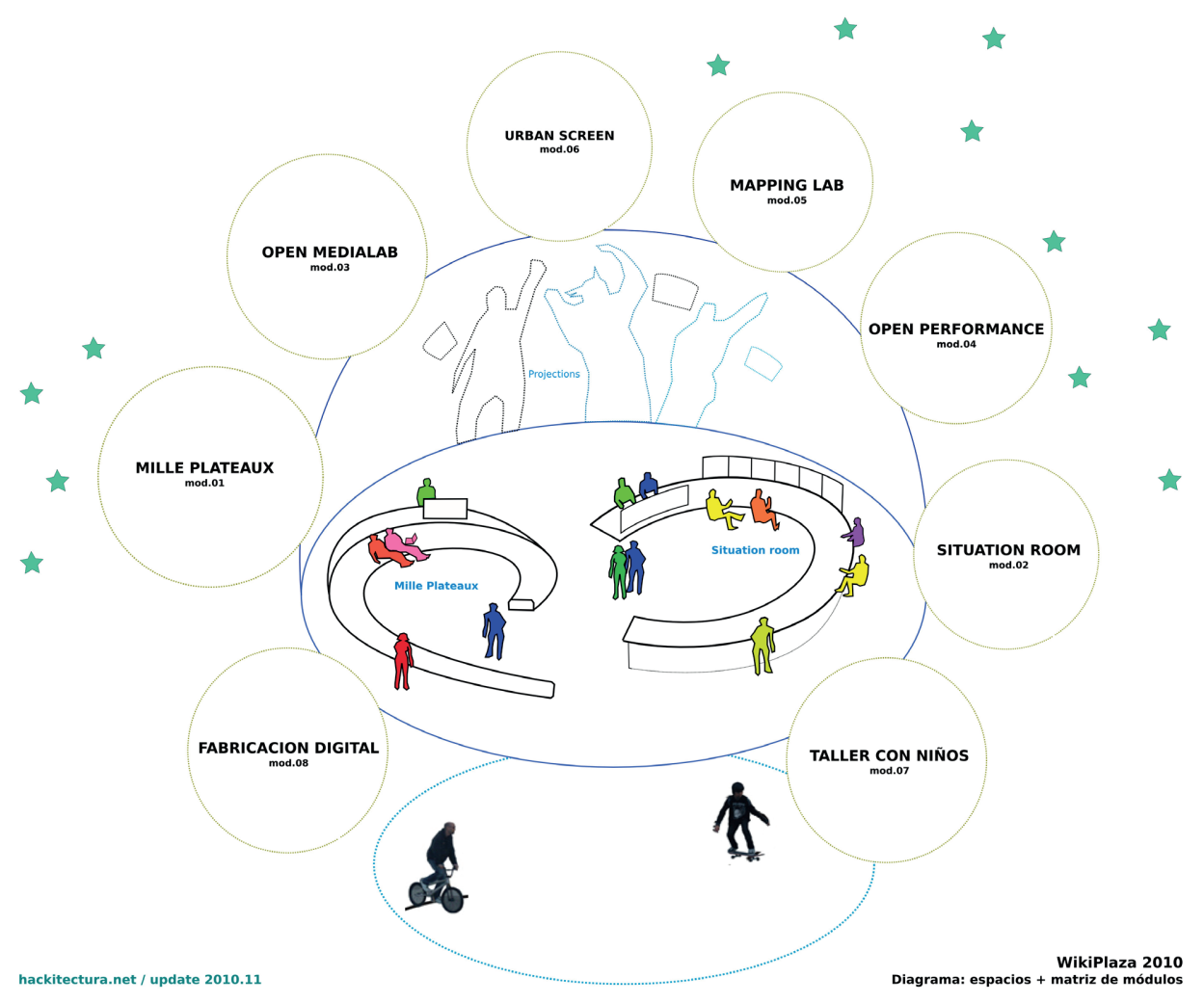

Figura 2.

Diagrama de

usos de la

Wikiplaza

Fuente: José

Pérez de Lama,

2009

\begin{abstract}
Para mí, el proyecto W4B fue sensacional, excelente. Totalmente vanguardista, sacando a colación un lugar de Sevilla de una manera muy particular, con las visitas a las ruinas del pabellón de Europa, el modelo tridimensional... La pena es que el proyecto no se haya visto un poco más, con muchas capas diferentes. Desde el arte se puede hacer crítica pese a partir de financiación privada (como la BIACS). Era una buena oportunidad.
\end{abstract}

$\mathrm{Al}$ contexto de crisis generalizada se le sumó un impago por parte de la BIACS que fue muy duro para una iniciativa de pequeña escala como Hackitectura. net, empujándoles al cierre del colectivo. A modo de epílogo se realizó el proyecto Mapping the Commons, reconocido con el I Premio Elinor Ostrom a la investigación e intervención social vinculada a bienes comunes por la Universidad de Buenos Aires. Realizado en Atenas y Estambul, concreta algunos apartados de la cartografía táctica investigados en proyectos previos: 
Mapping the Commons tiene un mucho de laboratorio. Siguiendo la trilogía de Hardt y Negri甲, si los Commons son esa hipótesis para cuestionar el sistema actual que es un desastre, vamos a evaluarlo en la ciudad de Atenas. Seguimos explorando maneras de generar el conocimiento donde se mezclan mapas, imágenes, textos, proyecciones.

\section{MOMENTO ACTUAL}

A modo de síntesis, concluye PDS:

Generamos situaciones muy experimentales que tenían que ver con cuestiones políticas, de espacio público y con la experimentación con las tecnologías. Por eso decíamos en la época que estábamos influenciados por el movimiento hacker, el situacionismo y la ciencia ficción. [...] Otra gente, con los pies más en la tierra, hubiera sido inimaginable. Se generaban esas relaciones orgánicas y rizomáticas que al final hacía que se juntara gente con mucho talento y muchos recursos en una estación abandonada del AVE o entre las dos orillas del estrecho. Era algo contingente con apariencia de película cyberpunk.

Añade JPL sobre las diferencias entre el contexto actual y el que se daba en aquella época: "Una cosa muy chula es que trabajábamos en el tiki-wiki. En aquella época valorábamos el trabajar online. Ahora intento evitar el smartphone, pero entonces estaba todo el día conectándome donde podía”.

A nivel personal, la trayectoria de cada componente ha profundizado en áreas de trabajo de la propia Hackitectura.net, con mayor o menor cercanía según la perspectiva desde la que se mire. Pablo de Soto, tras sus investigaciones en Fukushima, el parque Gezi en Estambul o sus diferentes trabajos en Sudamérica ${ }^{10}$ está actualmente afincado en Brasil, donde el movimiento reaccionario institucional, a partir de la elección de Bolsonaro en el Gobierno, censura las investigaciones de carácter activista como el mapeado sobre el asesinato de Marielle Franco:

El contexto ahora es duro. Las situation rooms de ahora son las que hace Facebook para las elecciones. La tecnopolítica de la extrema derecha es la que está ahora utilizando de manera antiética e ilegal las cajas negras tecnológicas (tipo Cambridge Analytics). La hackitectura sería cómo pensar ágoras para combatir esas fake news y traer discursos de paz. Es un momento más de retaguardia que de vanguardia [...] El mundo se ha tornado más distópico. Todo va alrededor de tu perfil personal. Antes no era así. Internet como lugar de trabajo, como las wikis, ha evolucionado a una internet básicamente de exposición. Nuestra web era un tiki-wiki con galería de imágenes, espacios de trabajo... Ha habido un cambio radical en la forma de usar internet. El funcionamiento de las búsquedas ha

9 La referencia que usa PDS y que usaron como base de los talleres Mapping the Commons es Commonwealth (Hardt, Michael \& Negri, Toni, 2009, Harvard University Press).

10 Para más información sobre los trabajos de Pablo de Soto, visitar su web personal: https:// pablodesoto.org/ 
cambiado completamente con Facebook, Google... Se ha perdido la autonomía de los medios. Antes eran minoritarios, pero con un cierto público. Ahora el ecosistema de información está mucho más centralizado. Se ha perdido esa diversidad más friki y heterogénea de esa internet de los 2000.

José Pérez de Lama ha dirigido uno de los proyectos claves, Fab Lab Sevilla ${ }^{11}$, en la implantación de la fabricación digital en España consiguiendo que uno de los nodos de mayor relevancia se encontrara en la Universidad de Sevilla. Frente a otros modelos, ha conseguido priorizar la democratización en la entrada a estas tecnologías por parte del alumnado y su extensión más allá de la universidad. Sin embargo, no es optimista con su evolución:

Hay un paralelismo entre la evolución del contexto que vivimos en Hackitectura con la trayectoria de los movimientos makers, fablabs y demás relacionados con la precariedad laboral. Exceptuando alguna parte de hardware libre (Arduino; las FPGA; RepRaps), la parte más experimental / política se la ha comido la innovación, las startups, las incubadoras de empresas.

Probablemente por su mayor implicación técnica, Sergio Moreno ${ }^{12}$ puede haber mantenido una continuidad más lineal respecto a la trayectoria de Hackitectura.net, por ejemplo, en materia de streaming:

En el proyecto Cimbra entre Cádiz y Cartagena de Indias [2012] puedes encontrar tecnologías, intervención en espacio, ocupación en el espacio, redes libres... sin limitaciones. El último proyecto en torno al streaming fue el Concierto de Jazz Distribuido que hicimos con el CICUS [en el] 2015. El concierto en streaming es lo más complicado de realizar. Cuando se emite vídeo y audio por internet hay una latencia. Estuvimos haciendo unos cálculos contando con fibra óptica y vimos que a lo mejor podíamos conseguir una latencia que quizá podría ser viable. La imagen da igual que vaya con algo de retraso porque el ojo está acostumbrado, pero para tocar un músico en un sitio y otro músico en otro, la latencia del audio tiene estar por debajo de un umbral (tres milisegundos) porque si no, se desincroniza. Por eso en las orquestas hay un director, para que no se descuadre toda la película. Utilizamos Gstreamer y unas tarjetas capturadoras de baja latencia y conseguimos una latencia suficientemente baja con cable de red a pocos metros. Ya daba igual lo que extendiéramos el cable de red con fibra óptica y ya daba igual cuántos metros de distancia porque la fibra tiene muy poca latencia. Pierde casi todo en la captura del sonido. Conseguimos una latencia mínima. Consumía mucho ancho de banda, pero funcionaba.

11 Para más información sobre Fab Lab Sevilla, actualmente dirigido por Enrique Vázquez, con la colaboración de Juan Carlos Pérez Juidías: http://fablabsevilla.us.es

12 Los trabajos de Sergio Moreno los sigue realizando en la cooperativa WWB y de la que forman parte antiguos colaboradores de Hackitectura.net como Misael Rodríguez o Alejandro González. Para más info: https://wwb.cc/ 
A partir de esa investigación no hubo recursos ni interés por profundizar más allá: "Los streaming de Fran Ilich, de superlatencia y muy baja calidad, han acabado dando cosas que están más o menos concluidas con estos trabajos de telepresencia".

En la línea de los servidores, "colaborando con Maadix del equipo de Xnet desarrollamos una plataforma de software libre sobre Drupal donde automatizar el proceso de montar el servidor, las aplicaciones... Es el proyecto en el que estoy más metido". También Pablo cree que Maadix es un proyecto que mantiene una coherencia y continuidad respecto a la obra de Hackitectura:

El proyecto de Maadix poniendo el foco en la criptografía y en la protección de datos en la nube sin duda es uno de los proyectos clave en nuestro contexto. El momento es de proteger algunas cosas que se tenían. Nuestro mundo digital es un libro abierto para aquellos que tienen el poder. Una hackitectura protectora de nuestro yo digital sería una continuación de la hipótesis.

\section{REFERENCIAS}

De Soto, Pablo (2010). Situation Room. Diseñando un Prototipo de Sala de Situación Ciudadana. Barcelona: DPR-Barcelona.

Moreno, Sergio; Pérez de Lama, José y H. Andrade, Laura (2011). Wikiplaza. Request for comments. Barcelona: DPR-Barcelona.

Pérez de Lama, José (2000). Vanguardia angelino-alienígena de un oficio viejo y otro nuevo. Pasajes de arquitectura y crítica, (22), p. 30.

Pérez de Lama, José (2006). Devenires Cíborg. Arquitectura, urbanismo y redes de comunicación. Sevilla: Servicio de Publicaciones de la Universidad de Sevilla.

Varios Autores (2006). Fadaiat. Libertad de movimiento + Libertad de Conocimiento. Málaga: Centro de Ediciones de la Diputación de Málaga. 
